MECHANIKA W LOTNICTWIE

ML-XIX 2020

https://doi.org/10.15632/ML2020/327-345

\title{
ANALYSIS OF RESONANT PROPULSION FLAPPING WING MICRO AERIAL VEHICLE
}

\author{
Kun Feng, Krzysztof SiBILski \\ Warsaw University of Technology, Faculty of Power and Aviation Engineering \\ e-mail:krzysztof.sibilski@pw.edu.pl
}

\begin{abstract}
This article is concerned with the resonant property which is exhibited in insect flight, and analyzes how resonant propulsion works when implemented in powering a flapping wing micro aerial vehicle. This article is divided into three parts. In the first part, information regarding to insect flight, the resonant property, and flapping wing micro aerial vehicles are described. In the second part, mathematical models representing the micro aerial vehicle (basing on the model developed by Bolsman) are applied, simplified and built into simulation in MATLAB. Some interesting properties from the simulations are presented.
\end{abstract}

Keywords: FMAV, MAV, resonant propulsion, simulation

\section{Introduction}

Everything in nature is finite with regard to energy and space. Especially, all living creatures are optimized in their own way through nature selection. Today, with well-developed theory of aerodynamics, advanced computing, we still do not fully understand the nature of flying beings and subtle things which enhance their flight. We are continuously studying the nature, looking for more inspirations in order to improve our own flying machines.

Birds and insects, for example, can be very small in size and weight, yet they possess all the essential systems to fly. Each part of their body is essentially developed to fly consistently and to consume as little energy as possible. And these properties are crucial for the development of small autonomous air vehicles with all systems calling for optimization.

With the development of micro batteries and processors, increasingly smaller aircraft can be developed. The design of micro air vehicles or MAV started around 1990s, from studying insect body structures, organisms and fly patterns. Throughout the years, many projects were made, many steps were taken, yet an exact biological replica from an insect is still far beyond our reach; many things in this field are still waiting to be studied.

A micro aerial vehicle or MAV, as defined by DAPRA, is a type of an autonomous aircraft with the size limitation of $150 \mathrm{~mm}$ in any linear dimensions, designed for environments and situations with strict size restrictions. They can be small, yet very capable. MAVs can be divided into three types by their means of lift generation: fixed-wing, rotorcrafts and flapping-wing (FWMAV). The first two types are well developed and commercially very successful. Fixed wing MAVs exhibit clean aerodynamic properties, rotorcrafts the hovering ability, MAVs with flapping wing lift generation appear to be most natural, however, very few projects have been carried out.

Some researchers focused on size minimization, e.g. the Harvard Fly project, with the wingspan of $30 \mathrm{~mm}$ and total mass of $60 \mu \mathrm{g}$. The wing flapping motion is powered by a piezoelectric actuator and amplified by the compliant mechanism. Others concentrated on replicating the real insect flapping motion, like in the mechanism proposed in [4], to investigate kinematics and aerodynamic properties. Engineers also tried to find alternative ways to realize the flapping motion. The Vanderbilt FWMAV applied the resonant property for actuation used in such a 
way that the left and right wing could be flapping at different frequencies. The Bolsman project applied the resonant property as well. His model is implemented in this article, and more details are described in latter Sections.

\section{Biological inspiration}

In flapping flights, whether mechanical or biological, wings take the major part of the total inertia, which means that most of the energy is consumed by flapping motion. Minimization of the energy cost while realizing this flapping motion is the starting point of research.

Unlike birds and bats, insect wings are passive structures, which means that the wing shape does not actively change during flight, thus no active actuation system is needed on the wing platform. Also, the flight control and lift generation are realized together only by wing motions. Insects do not require tails in order to stabilize motion, which makes them truly inspirational when it comes to the FWMAV design.

The flight muscles are usually the best developed in the insect body and occupy most of the space in thorax. The total flight muscle mass must be greater than $12 \%$ of the body mass in order to support the weight of an insect in flight.

Insect wings are driven by thorax which connects the wings and muscles in an elastic way. Thorax-wing connection or mechanism can be categorized into two types, Direct Driving Mechanism (DDM), and Indirect Driving Mechanism (IDM). In the DDM, the wings are connected to thorax muscles at different points on the wing root. The wings are driven directly by elevation and depression of thorax muscles. When the thorax muscles elevate, the wings are lifted, and as thorax muscles depress, the wings are then deriven back to the previous position. This type of mechanism allows achieving a huge flapping angle and differential wing motion and control (Fig. 1).

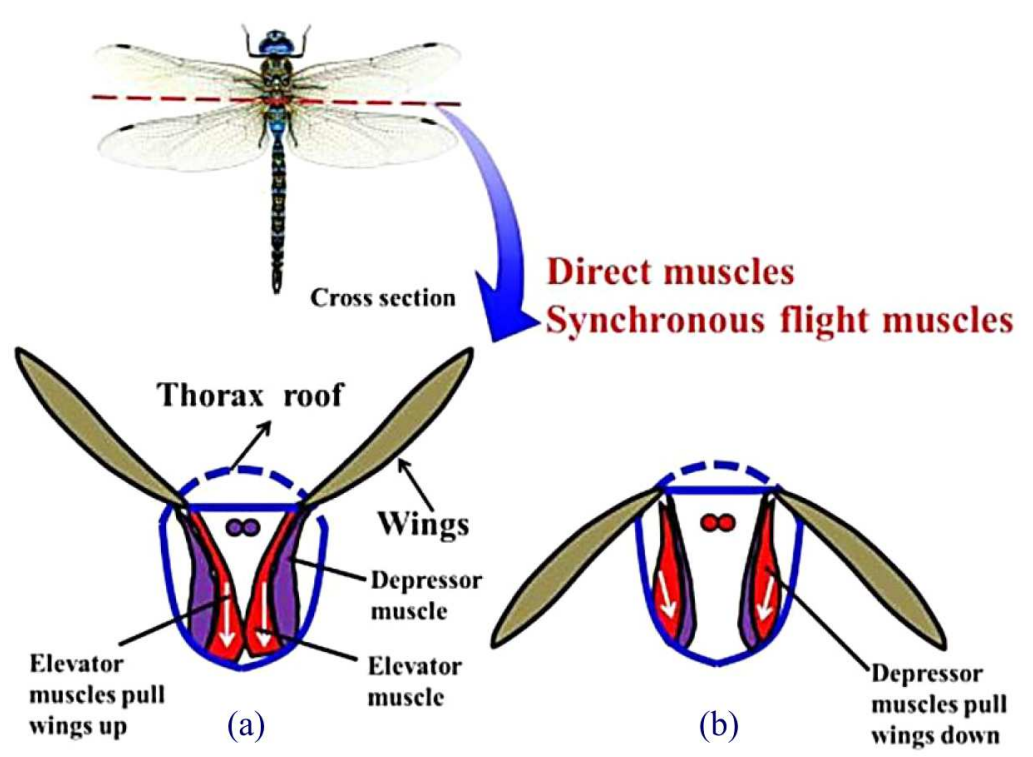

Rys. 1. Diagrammatic cross-section of insect Direct Driving Mechanism (DDM). Thorax mechanisms of direct muscles: (a) the wing is stroked up by contracting the elevator muscles, (b) the wing is stroked down by shortening the depressor muscles [8]

In the IDM (Fig. 2), the wing roots are linked to the upper and lower thorax shells, muscles are also connected to the muscle shells. As muscles elevate and depress, the thorax is moved up and down, the wing roots are connected to the thorax shell at different points, so as the 
thorax shell oscillates, the wings flap up and down, consequently, forming a type of a compliant mechanism. This type of the mechanism enables huge flapping frequency.

(a)

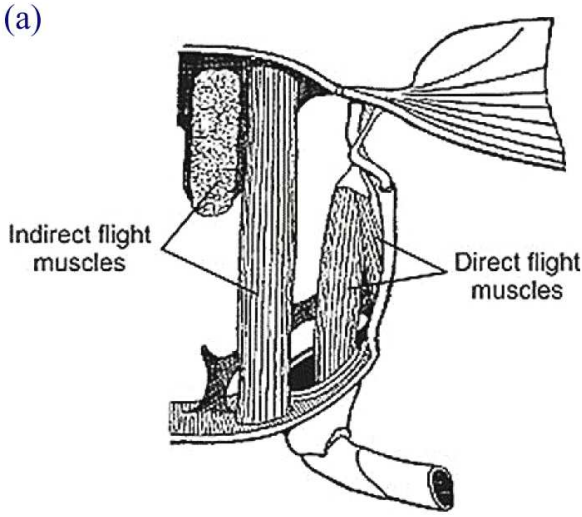

(b)

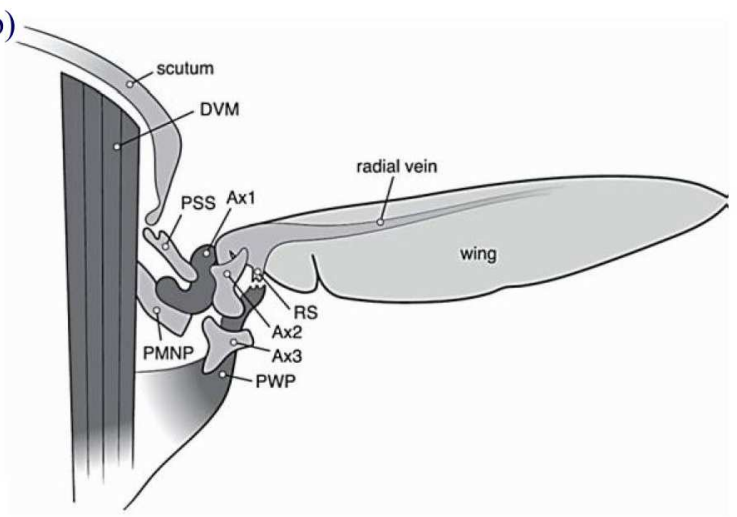

Rys. 2. (a) Cross-section of a generalized insect thorax. Indirect flight muscles change the shape of the thoracic box. Direct flight muscles are connectd directly to the wing insertion [6]; (b) transverse section of the thorax of a fly in rear view showing some elements of the complex wing hinge of the fly consisting of ridges and protrusions on the thorax and a number of hardened plates of cuticle (sclerites) between the body (thorax) and the wing root. The basalare sclerite (not shown) is positioned anterior of the first axillary sclerite (Ax1). The indicated structures are the dorso-ventral power muscle (DVM), pleural wing process (PWP), post-medial notal process (PMNP), parascutal shelf (PSS), axial wing sclerites (Ax1, Ax2, Ax3) and radial stop (RS). Redrawn and modified from [13]

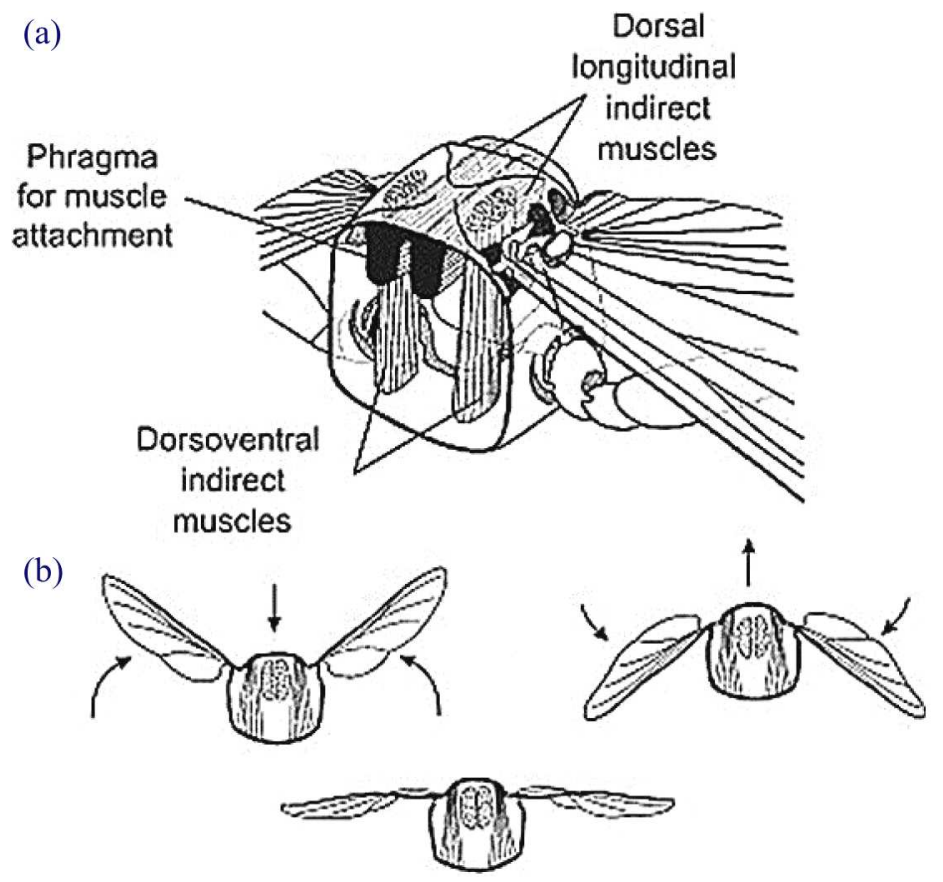

Rys. 3. The mechanism of wing movement: (a) the indirect dorsal longitudinal muscles attach to the phragmata at either end of the segment causing it to shorten and move the wings downwards. The indirect dorsoventral muscles move the tergum downward with contraction causing the wings to move upwards; (b) wing movements as a result of changes in thoracic conformation [6]

There are three general categories of muscles that power insect flight: direct, indirect, and accessory. Direct flight muscles, consisting of the basalar and subalar muscles, are inserted directly at the base of the wing and provide power for the downstroke in more primitive insects, and also affect wing pronation and supination (Fig. 4). Another direct muscle, the third axillary 
muscle, is inserted in the third axillary sclerite. It affects wing supination and is also responsible for wing flexion against the body wall when the wings are at rest. In contrast, the indirect flight muscles move the wings indirectly by changing the conformation of the thorax. These muscles include the dorsoventral group that extends from the tergum to the sternum. Because of the structural relationships among the sclerites that make up the thorax, in all insects, when the muscles pull the tergum down they indirectly cause the wings to raise and produce the upstroke (Fig. 4). Another indirect group, the dorsal longitudinal muscles, are attached longitudinally between the two phragmata of each wing-bearing segment. When they contract, they shorten the segment and cause the tergum to elevate. In more advanced insects, this deformation of the notum by the dorsal longitudinal muscles produces the power stroke by depressing the wing. These muscles are reduced in more primitive insects which use instead the direct muscles for the downstroke (Fig. 5).

Primitive wing control

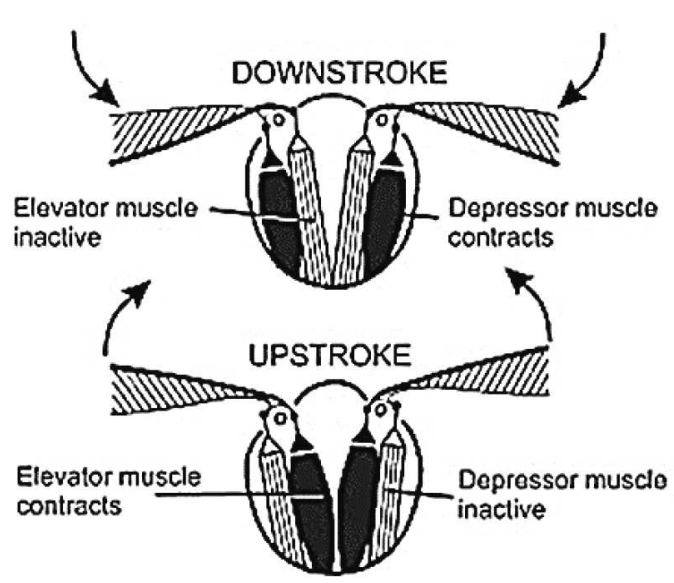

Advanced wing control

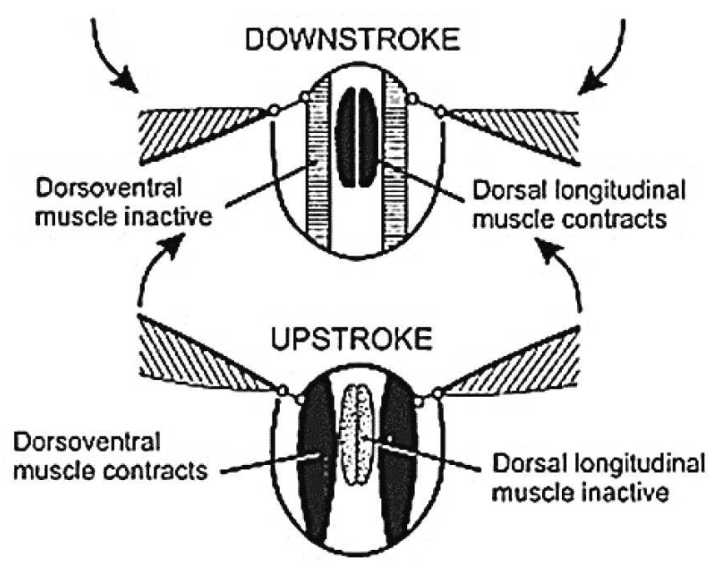

Rys. 4. Differences between more primitive wing control by direct flight muscles (left) and more advanced wing control by indirect flight muscles (right) [6]

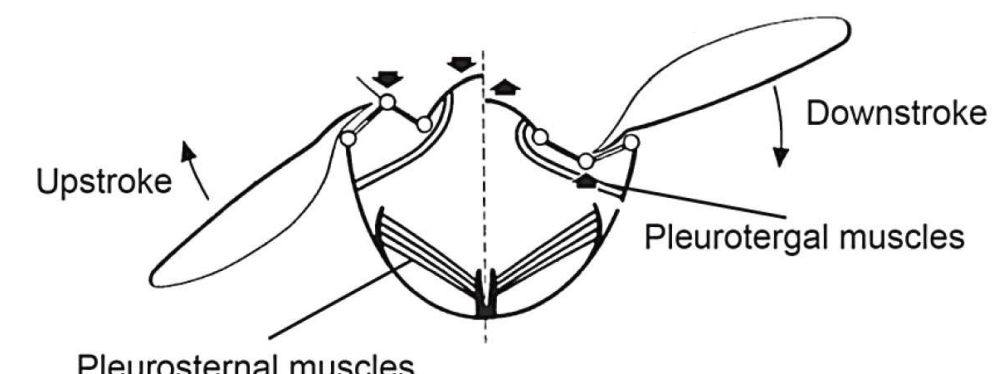

Rys. 5. Pleurosternal and pleurotergal muscles are accessory muscles that change the shape of the thorax and modulate the output of indirect flight muscles [6]

There are also accessory muscles that are inserted into the thorax and influence its mechanical conformation. For example, the pleurosternal and pleurotergal muscles modulate the power output and the nature of the wing beat by changing the orientation of the thoracic plates and resonance of the thorax (Fig. 5).

From the engineering point of view, in IDM, the thorax shell acts as an elastic element between the actuator and wings. When this elastic element is introduced, some energy can be stored from the wing movement, so less energy is required to drive the subsequent wing motion, consequently, smaller muscles are needed. This is the resonant property in insect flight, or more specially, in insect flight with the IDM. 
To introduce this resonant property in a mechanical structure does not necessarily mean that the system will work in the natural frequency. However, as the vibrating frequency tends to the natural frequency, due to the storage of elastic energy in the structure, small periodic forces will be able to provide large oscillations. So, with a carefully designed compliant mechanism, the resonant property can amplify motion or achieve full propulsion.

\section{Aerodynamics and kinematics}

Insect flight aerodynamics differs hugely from normal aerodynamic analysis. In insect flights, the Reynolds number can be as little as 10, the beating frequency can go as high as $200 \mathrm{~Hz}$, the standard terminology does not come into play. The quasi steady method proposed in [1] is applied in this paper in order to understand the unsteady aerodynamics of the insect flight and formulate it properly. Three aerodynamic mechanisms are to be explained shortly.

First of all, the leading edge vortex, LEV, develops on the translating wing as a result of the increasing angle of attack or AOA. As the AOA increases, the incoming flow over the wing does not follow the wing chord path as it should be, instead, it sheds away from the wing at the leading edge. And as it sheds away, the flow becomes a vortex and creates a separation zone. Then, the vortex goes back and reattaches to the wing before reaching the trailing edge, The LEV shown in Fig. 6 has an velocity vector in the span-wise direction, so the LEV spirals down from the root to tip, attaches to a major part of the wing, and the Kutta-Jukowski condition holds in this case.

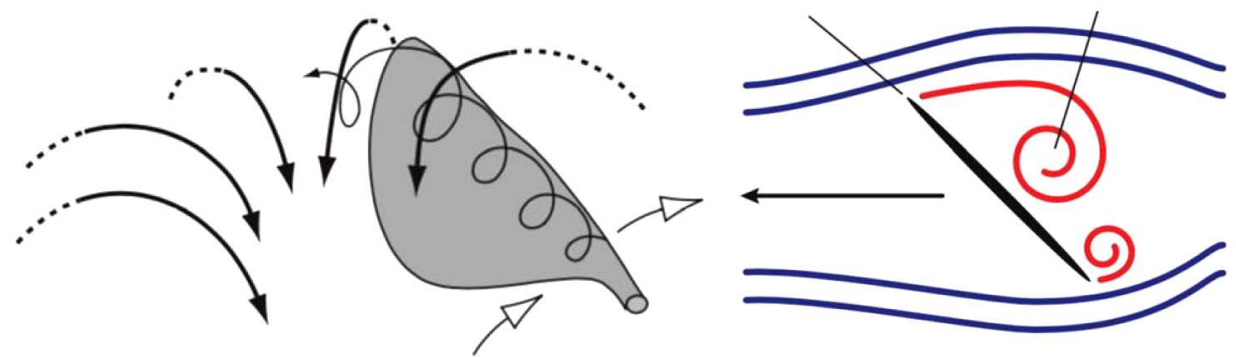

Rys. 6. Leading edge vortex spirals to the wing tip [3]

At the end of every stroke, a change in phase happens as to remain positive angles of attack during both upstroke and downstroke. In [3], the author found that the phase shift had significant aerodynamic importance to the total creation of lift. During the phase shift, the wing rotates about a span-wise axis, the flow around the wing deviates from the Kutta-Jukowski condition, which causes a dynamic gradient at the trailing edge of the wing, leading to shear. However, fluid viscosity is resistant to shear, so circulation is generated around the wing to counteract the rotational effect and re-establish the Kutta-Jukowski condition.

This can be explained by another way, by assuming the flow under complete inviscid condition. Under such a condition, the flow has the minimum deflection at the leading and trailing edge, so the rear stagnation point is shifted from the tip to the upper trailing edge, making the flow to behave like in Fig. 7. However, in order to maintain this shape, a huge velocity gradient turns the flow very sharply at the tip.

The flow over the wing is shed into the LEV and trailing edge vortex, and the vortices detach from the wing at the end of the stroke. The vortices are with a velocity component normal to the wing. After the phase shift, wing AOA changes back to positive in the subsequent stroke, and "capture" the vortices. The vortices have an enhanced velocity and acceleration field, thus the pick of lift generation appears immediately after the reverse stroke. And this is the third mechanism, the wing-wake reaction. 


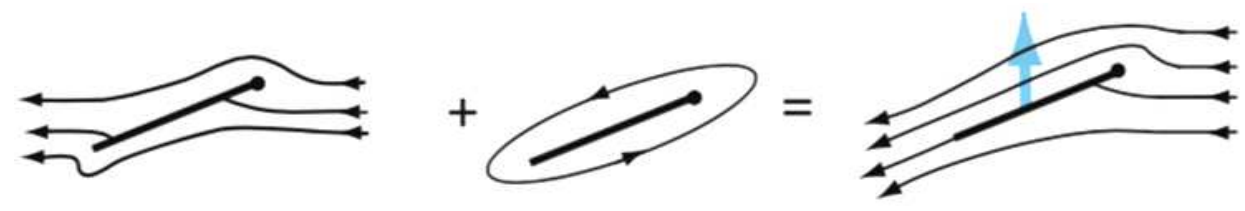

Rys. 7. Rotational circulation [11]

There are still other mechanisms which may explain lift generation and enhancement in insect flight. These three mechanisms are later to be used for lift computation. After explaining the aerodynamic phenomenon, the wing modeling can be done.

The rigid wing motion can be described by three angles, the main flapping angle $\phi$, the wing pitching angle $\eta$, and the out of plane angle $\theta$ (Fig. 8).
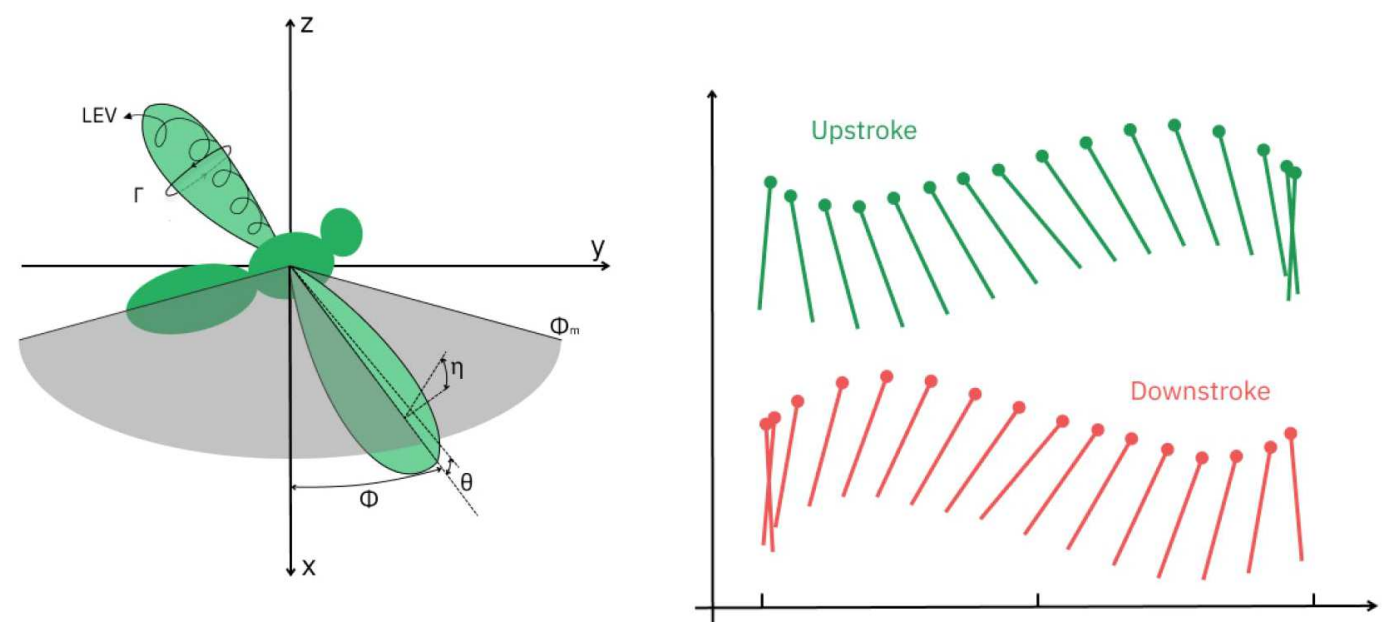

Rys. 8. Insect wing motion. Schematic and aerodynamics (left), upstroke and downstroke (right)

As the insect wing flaps up and down, it leaves a trace of a plane behind, called the stroke plane, shown in Fig. 8. It can be compared with the helicopter rotor plane from blades rotation. In the stroke plane, the angle between the wing and the $x$ axis is the main flapping angle $\phi$ which is limited by the maximum flapping angle $\phi_{m}$. The flapping angle is the most characteristic motion of the insect flight. The pitching angle $\eta$ is defined as the angle between the wing and the stroke plane, it is not the angle of attack, it is influenced by the phase shift and inflow angle, some offset needs to be introduced to obtain the AOA. Last but not least, the out of plane angle $\theta$, or heaving motion, describes deviation of the wing from the stroke plane. In the right part of Fig. 8, the upstroke, downstroke and two phase shifts and illustrated.

\section{Model of micro aerial vehicle with flapping wings}

Before simulation, a short test was done. Several important features of the FWMAV design from [2] are stated here for the analysis. First of all, to design a thorax like structure, a bearing element in order to effectively store the potential energy and, at the same time, withstand oscillation in form of a unidirectional carbon fiber ring was chosen. Then, to connect the wings and achieve the passive wing pitching, elastic hinges between wing and the ring were designed, which can be seen in Fig. 9 .

After acknowledging basic concepts, building a practical mathematical model is the next step. The general problem now can be treated as a vibration problem. This Section is strongly based on the method used in [2]. 

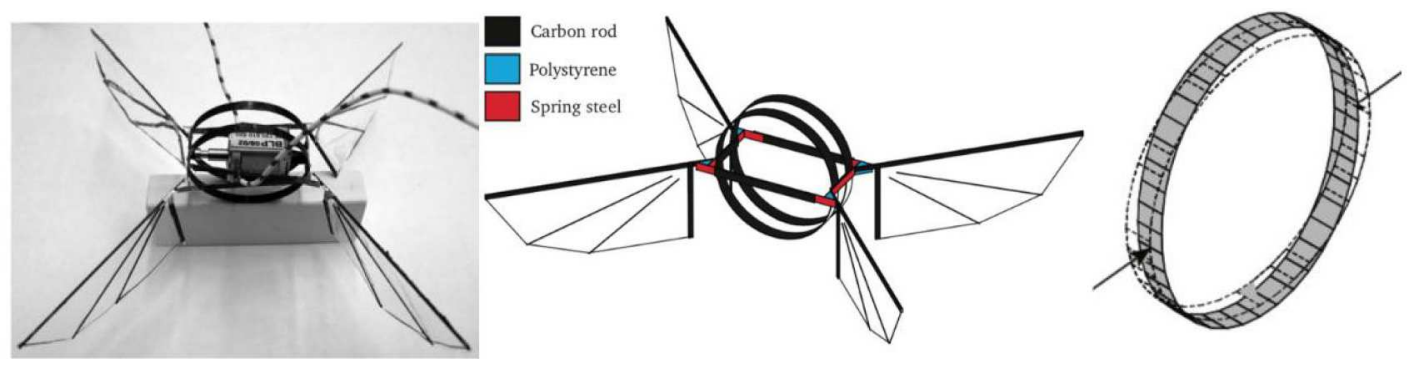

Rys. 9. FMAV design by Bolsman (left), layout and material (center), deformation of the center elastic ring structure (right) [2]

To simplify the model, an abstraction of the thorax-wing system is used and shown in Fig. 10. A half view is presented there. The intersection of the horizontal line and the dashed line is the rotation point of the wing. It represents the wing root. On its left side there is the body, on right one, the wing. The system is viewed as having only one degree of freedom represented by the wing sweeping angle $\phi$. There are only two points of masses, $m_{b}$ and $m_{w}$ which represent the total inertial properties of the system. The spring constant $k$ shows the overall stiffness properties of the system and is seen as a torsional spring. The aerodynamic force attached to the wing is $F_{d}$ and is always acting in the opposite direction to the motion. $L$ is length between the wing attachment point and the aerodynamic center.
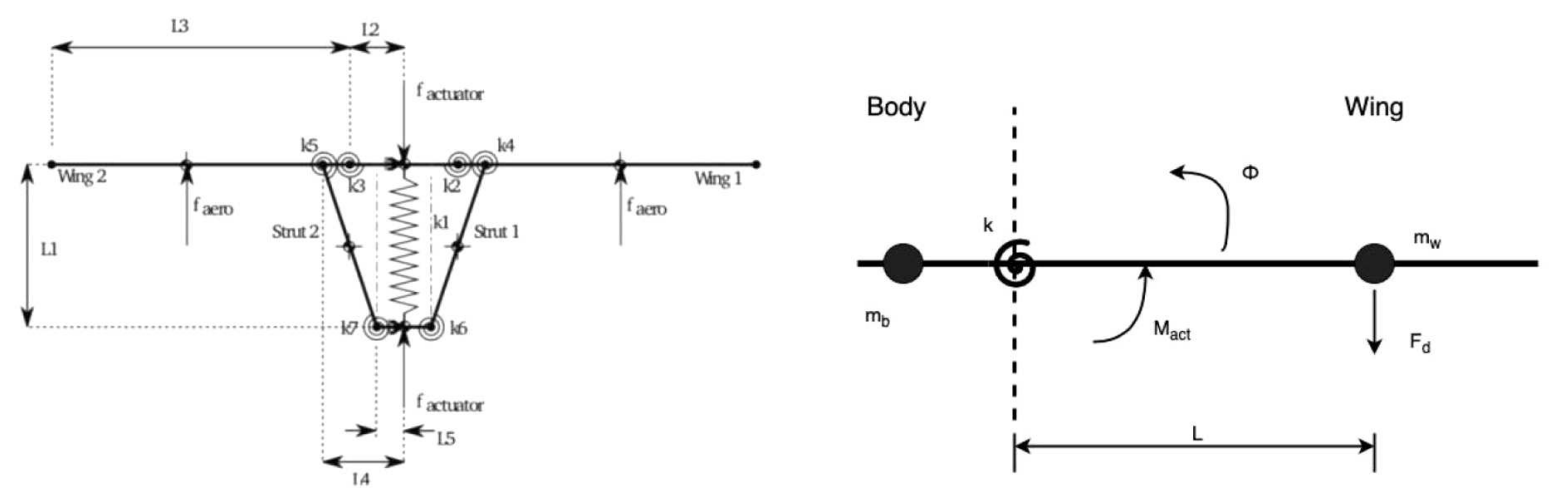

Rys. 10. Concept and linear dimensions of resonant flapping wings propulsion (right) and a schematic representation of the wing-thorax system (left) [2]

As a spring-mass damping system, the equation of motion can be written as

$$
I \ddot{\phi}+k \phi=M_{a c t}(\dot{\phi})-M_{a d}(\dot{\phi})
$$

where $I$ stands for the equivalent inertia of the whole system including the body and both wings, $k$ represents the spring constant of the generalized stiffness of the system, $M_{a c t}(\dot{\phi})$ is the applied moment acting on the root of the wing, $M_{a d}(\dot{\phi})$ shows the moment produced by the aerodynamic force, $\dot{\phi}$ is the velocity term of sweeping motion $\phi, \ddot{\phi}$ is the acceleration term of sweeping motion $\phi$.

A simple hormonic motion is assumed based on the simple harmonic sweeping motion which takes place in real insects with flapping wings, so the equations of the flapping motion and its derivatives can be written as

$$
\phi=A \sin \omega t \quad \dot{\phi}=A \omega \cos \omega t \quad \ddot{\phi}=-A \omega^{2} \sin \omega t
$$

The cycle of this harmonic motion is $T$, which can be computed from: $T=2 \pi / \omega$, and the maximum amplitude of the wing sweeping motion $A$, or the harmonic term, is restricted by the 
FWMAV setup or flapping properties of the real insect. It is unknown at this point, because the motion is not taken from anywhere, it will be a result of vibrational motion to be computed and analyzed in subsequent Sections.

The body mass $m_{w}$ and the wing mass $m_{b}$ represent the generalized inertia of the system. The body mass $m_{b}$ is much greater than the wing mass $m_{w}$, so the body is assumed as stationary, then the inertial of the whole system $I$ can be treated as $I=m_{w} L^{2}$. The wing inertia dominates the total effective inertia.

Now, the equation of motion can be simplified

$$
M_{a c t}(\dot{\phi})=I \ddot{\phi}+k \phi+M_{a d}(\dot{\phi})=A \sin \omega t\left(k-m_{w} \omega^{2} L^{2}\right)+M_{a d}(\dot{\phi})
$$

At this point, in order to highlight the resonance properties of the system, the stiffness term $k$ can be computed from $k=m_{w} L^{2} \omega_{0}^{2}$, simplifying the equation again

$$
M_{a c t}(\dot{\phi})=A \sin (\omega t) m_{w} L^{2}\left(\omega_{0}^{2}-\omega^{2}\right)+M_{a d}(\dot{\phi})
$$

At this point, the aerodynamic forces are calculated, and according to [3], they can be approximated as

$$
M_{a d}(\dot{\phi})=C_{d, c} L^{3} \dot{\phi}^{2} \quad F_{l}=C_{l, c} L^{2} \dot{\phi}^{2}
$$

where $C_{d, c}$ is the corrected drag coefficient and can be written as $C_{d, c}=C_{D} S \rho / 2, C_{l, c}$ is the corrected lift coefficient and can be written as $C_{l, c}=C_{L} S \rho / 2, S$ is the wing surface area, $\rho$ is density of the air, $C_{D}$ is the dimensionless drag coefficient of the wing, $C_{L}$ is the dimensionless lift coefficient of the wing. While introducing the lift force into the equation, because a large portion of the energy is consumed by wing flapping motion, also a limitation is to be defined: $\bar{F}_{l}=m_{t o t} g$, where $m_{t o t}$ is the total weight, $m_{t o t}=m_{w}+m_{b}$. To keep the system hovering

$$
\bar{F}_{l}=\frac{1}{T} \int_{t}^{t+T} F_{l} d t=\frac{1}{2} C_{l, c} L^{2} A^{2} \omega^{2}
$$

And in the final form

$$
M_{a c t}(\dot{\phi})=A m_{w} L^{2}\left(\omega_{0}^{2}-\omega^{2}\right) \sin \omega t+\frac{C_{d, c}}{C_{l, c}} L\left(m_{w}+m_{b}\right) g(1+\cos 2 \omega t)
$$

So, the total problem breaks down to one equation. On the right hand side of the final form, the inertial and stiffness properties are expressed in the first term. The second term presents aerodynamic properties. On the left hand side, the required actuator moment is presented as a function of the flapping velocity $\dot{\phi}$.

To start simulation, linear sizing is the first step as explained in the previous Section. The linear sizing data of concept 1 is directly taken, and then there will be minor changes only. A schematic representation of the model of resonant flapping wings propulsion is shown in Fig. 10. All major components, including the wing, struts, hinges are shown in the graph. The dashed line at the sides of the spring represent the elastic ring. This schematic presentation can be compared with Fig. 7 for better understanding.

Looking at Fig. 10, $L_{1}$ is the ring diameter, $L_{2}$ is the hinge length, $L_{3}$ is the half wingspan, $L_{4}$ indicates where the struts are connected to the wing, and $L_{5}$ is the ring width. Additionally, $h_{r}$, which is the ring thickness, is also added. Additionally, $\bar{c}$ stands for the mean chord length, $h_{w}$ for wing thickness and, finally, $b_{r}$ the ring width.

In order to further investigate and exploit the resonant property, the general stiffness term and the frequency of the system need to be determined. In Fig. 11, there is a presentation about how the ring structure behave under such loads. 


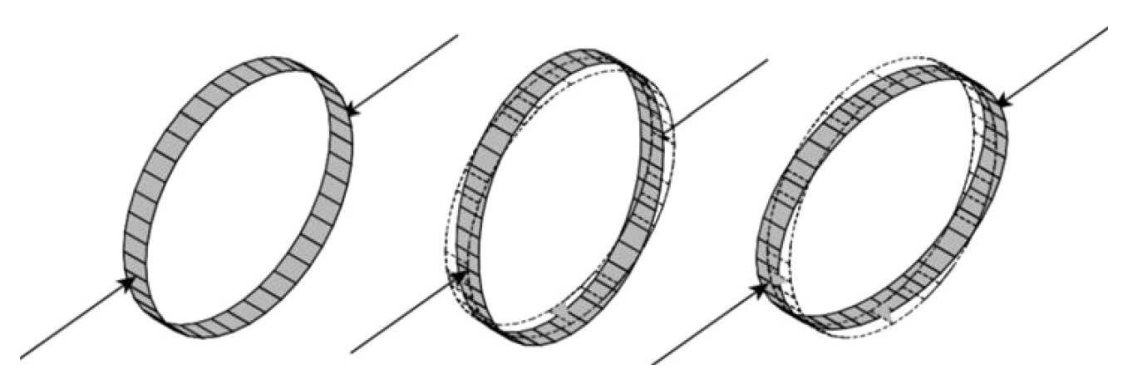

Rys. 11. A representation of how the ring structure is used as a spring [2]

The transmission ratio can be defined as the sensitivity of the design through the input-output relation, which is also a way to check the sizing correctness. The formula is presented below

$$
T=\frac{\phi}{u}
$$

where $\phi$ represents the flapping motion output, in degrees, $u$ stands for the actuator linear input or deflection of the ring in the radial direction, expressed in millimeters.

According to the schematics in Fig. 10, the transmission function can be expressed as

$$
u=\left(L_{4}-L_{2}\right) \cos \phi \quad \text { then } \quad \phi=\cos ^{-1} \frac{u}{L_{4}-L_{2}}
$$

This is a simplified relationship between the input and the output without considering the influence of elasticity, so the relation will be rather linear. The original [2] and other values of $L_{4}$ and $L_{2}$ are chosen, calculated and compared.

First of all, the ring volume is calculated to be $175.84 \mathrm{~mm}^{3}$. Then, for the ring, several standard materials which exhibit both good elastic and strength properties are chosen. They are from a typical stainless steel to the unidirectional carbon fiber. The equation for calculating the ring stiffness has the following form

$$
K=\frac{E I_{r}}{0.149 L_{1}^{3}}
$$

where $E$ stands for Young's modulus of the selected material, $I_{r}$ is the moment of inertia of the elastic ring. The inertia of the ring can be simply calculated from the equation: $I_{r}=m_{r}\left(L_{1} / 2\right)^{2}$ and the ring stiffness equation can be simplified to [2]

$$
K=0.005817610552 \frac{E m_{r}}{0.596 L_{1}}
$$

The ring resonant frequency $k$ can be calculated as

$$
k=m_{t o t} \omega_{0}^{2}
$$

According to [2], the desired frequency is between 20 to $30 \mathrm{~Hz}$, so the desired value of ring stiffness should be from $200 \mathrm{~N} / \mathrm{m}$ to $400 \mathrm{~N} / \mathrm{m}$. In this case, the only option for the material choice here is the unidirectional carbon fiber.

\section{Response analysis}

In order to examine numerical data, the transmission ratio and applicability of the model, also to investigate how the selected materials behave, several analyses have been made with the help of MATLAB program. 
The equation of simple harmonic motion has been established. In this equation, $F(t)$ represents the external force which in this case is only the aerodynamic force

$$
F(t)=\frac{C_{d, c}}{C_{l, c}} m_{t o t} g(1+\cos 2 \omega t)
$$

Introducing an equation

$$
-m_{t o t} A \omega^{2} \sin \omega t+k A \sin \omega t=\frac{C_{d, c}}{C_{l, c}} m_{t o t} g(1+\cos 2 \omega t)
$$

thus, the harmonic simple response $A$ can be calculated

$$
A=\frac{\frac{C_{d, c}}{C_{l, c}} m_{t o t} g(1+\cos 2 \omega t)}{k \sin \omega t-m_{t o t} \omega^{2} \sin \omega t}
$$

After obtaining the value of $A$, the motion $\phi=A \sin \omega t$ and the aerodynamic force $F(t)$ can be simply calculated with the help of MATLAB, and the results are quite promising. Two frequencies were chosen with the resultant output motion $\phi$, and aerodynamic forces are compared.

As the desired flapping frequency is around $20 \mathrm{~Hz}$ and $30 \mathrm{~Hz}$, so the time step was set as 0.001 , from 0 to $0.06 \mathrm{~s}$, in order to have a better view of the full flapping cycle (Figs. 12-15).

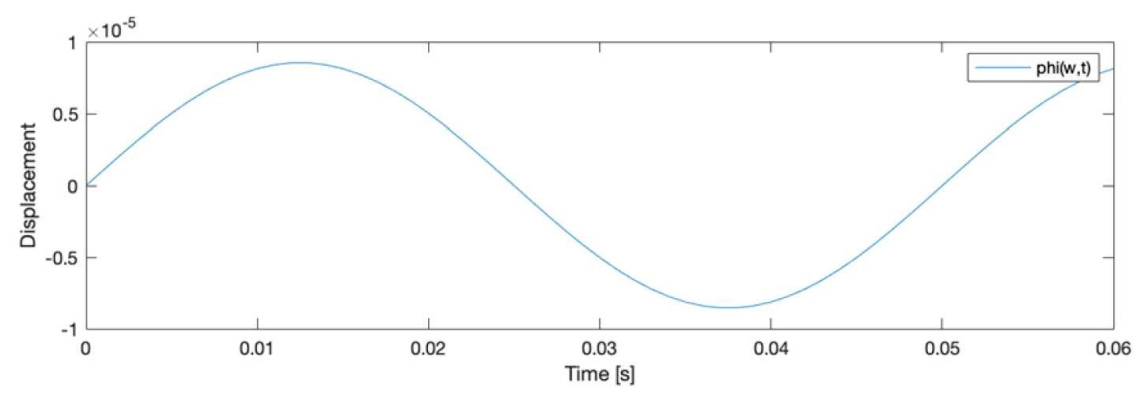

Rys. 12. System response at natural frequency $20 \mathrm{~Hz}$

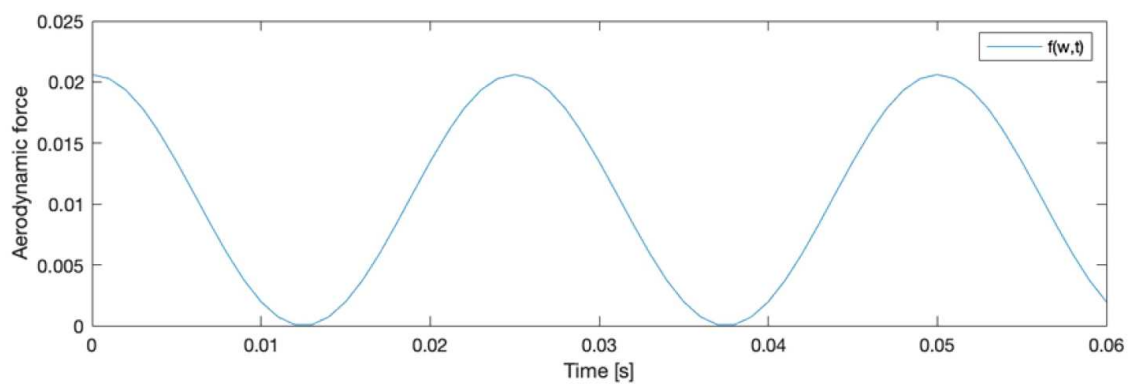

Rys. 13. Aerodynamic force at natural frequency $20 \mathrm{~Hz}$

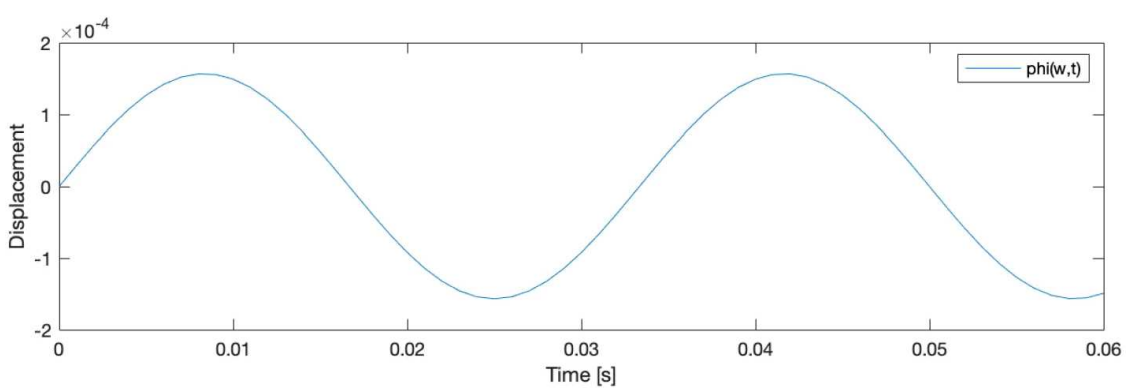

Rys. 14. System response at natural frequency $30 \mathrm{~Hz}$ 


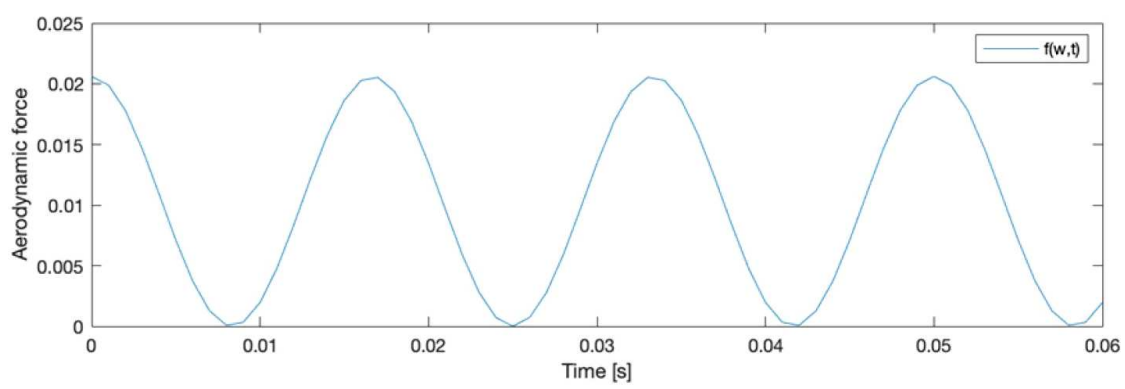

Rys. 15. Aerodynamic force at natural frequency $30 \mathrm{~Hz}$

Due to the simplified equation and inaccurate values of parameters, the specific output value of displacements and aerodynamic forces can also be incorrect. However, the catachrestic sinusoidal property holds and it provides quantitative information. The decrease of frequency by $10 \mathrm{~Hz}$ can result in a response decrement by one order. In the second comparison one observes the influence of stiffness. As the stiffness changes, the natural frequency of the system changes as well. Two tests were made and two stiffness values were investigated. The frequency however, was set to be at $98 \%$ of the natural frequency, making the response easier to compare (Figs. 16-19).

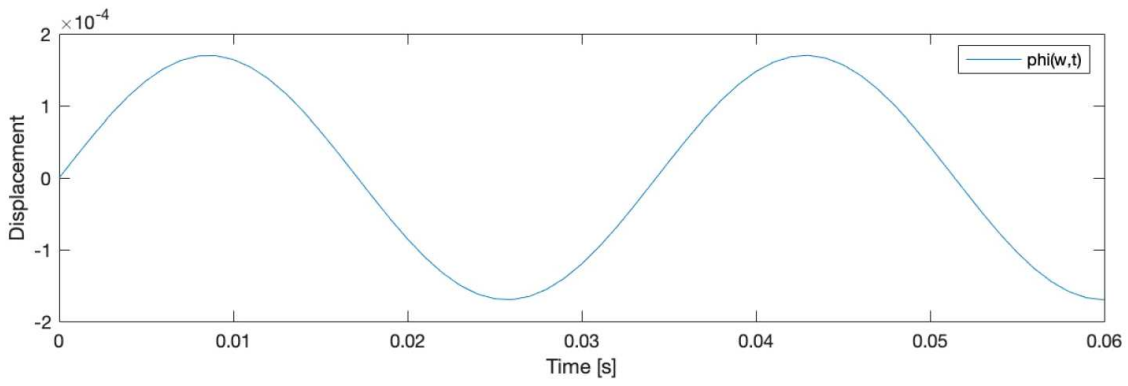

Rys. 16. System response at $k=200 \mathrm{~N} / \mathrm{m}$

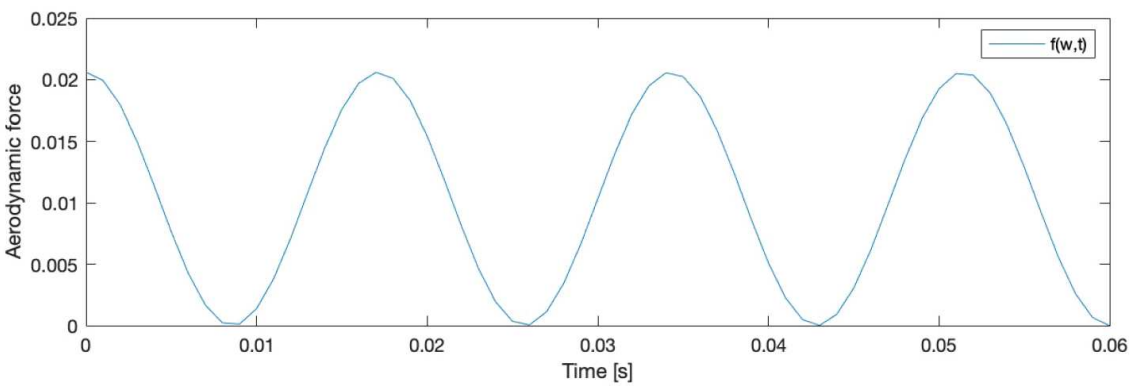

Rys. 17. Aerodynamic force at $k=200 \mathrm{~N} / \mathrm{m}$

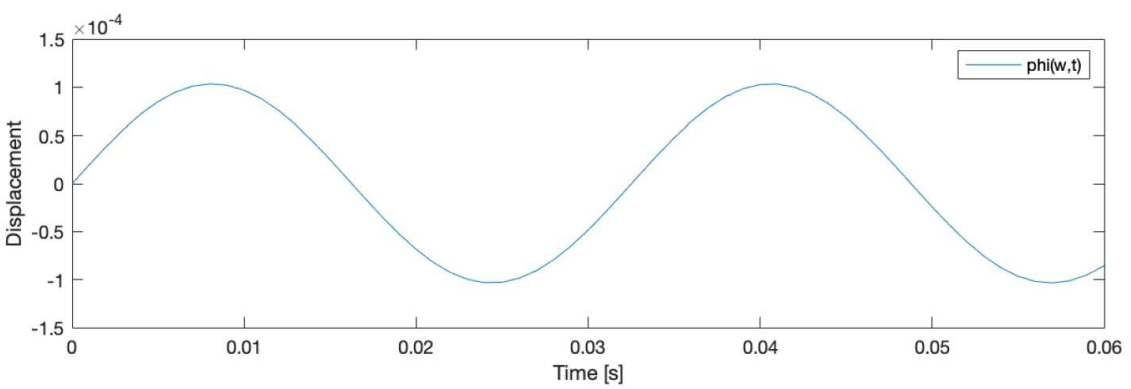

Rys. 18. System response at $k=400 \mathrm{~N} / \mathrm{m}$ 


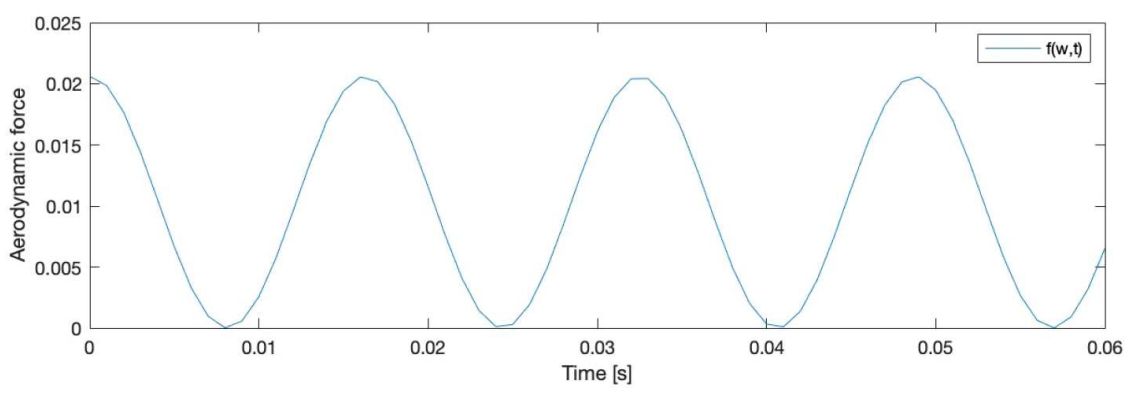

Rys. 19. Aerodynamic force at $k=400 \mathrm{~N} / \mathrm{m}$

The purpose of the frequency response is to find a steady state response for the sinusuodal input, which helps one to verify the frequency of the input signal over a certain range, and the resulting response can be studied.

For this system, specifically, the frequency response is a spectrum of vibration. To measure the frequency response, one needs the input force and the vibration response. However, at this point, the system is seen as vibrating freely, only influenced by aerodynamic forces with the acting force unknown.

The other thing is that the frequency response function is a three dimensional quantity. It is hard to represent generally, so a Bode diagram will be used, as shown in Fig. 20. The lower graph represents dynamic flexibility to frequency, and the upper one shows how the phase changes with respect to frequency. The frequency response analysis by the FRF analyzer gives really promising results.

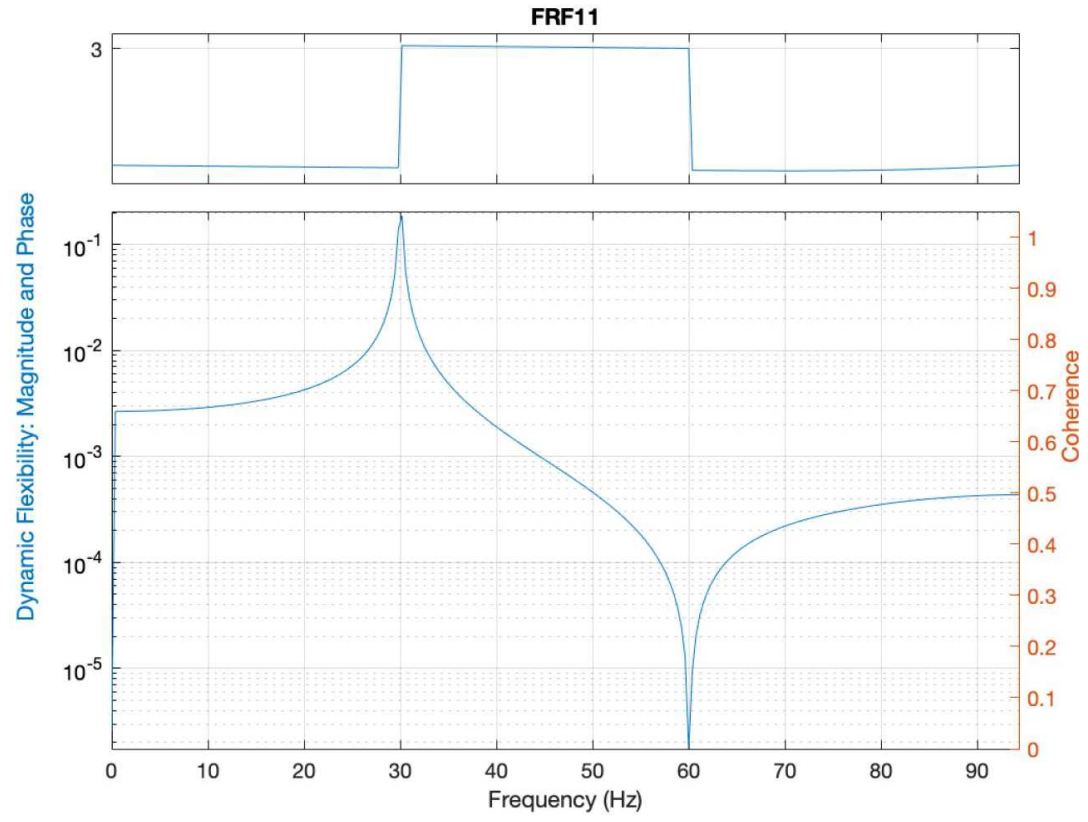

Rys. 20. Frequency response for the system using FRF analyzer

Looking from the results, it is clear that the system has a change of phase near the natural frequency $30 \mathrm{~Hz}$, however the change is rather stiff due to that the system is not damped. If the system is sufficiently damped, the phase change will appear much smoother. The transfer function for the system can be calculated. And with the help of Laplace transformation tables, the following are calculated

$$
I=m_{w} L \quad M_{a d}=2 \frac{C_{d, c}}{C_{l, c}} L_{w} m_{t o t} g \quad M_{a c t}(t)=L_{w \text { root }} F_{a} \sin \omega t
$$


Then, using the Laplace transformation, assuming that the initial condition are $\dot{\phi}(0)=0$ and $\dot{\phi}(0)=0$, the transfer function can be calculated as

$$
G(s)=\frac{\phi}{u}=\frac{\phi}{F_{a} \sin \omega t}=\frac{L_{w r o o t}}{s^{2}\left(I+M_{d}\right)+k}=\frac{L_{w \text { root }}}{s^{2}\left(m_{w} L+2 \frac{C_{d, c}}{C_{l, c}} L_{2} m_{t o t} g\right)+k}
$$

Taking the numerical data, the final version can be written as

$$
G(s)=\frac{0.01}{0.001236204 s^{2}+381.1}
$$

With the help of MATLAB, we can generate the Bode plot for this transfer function, see Fig. 21.

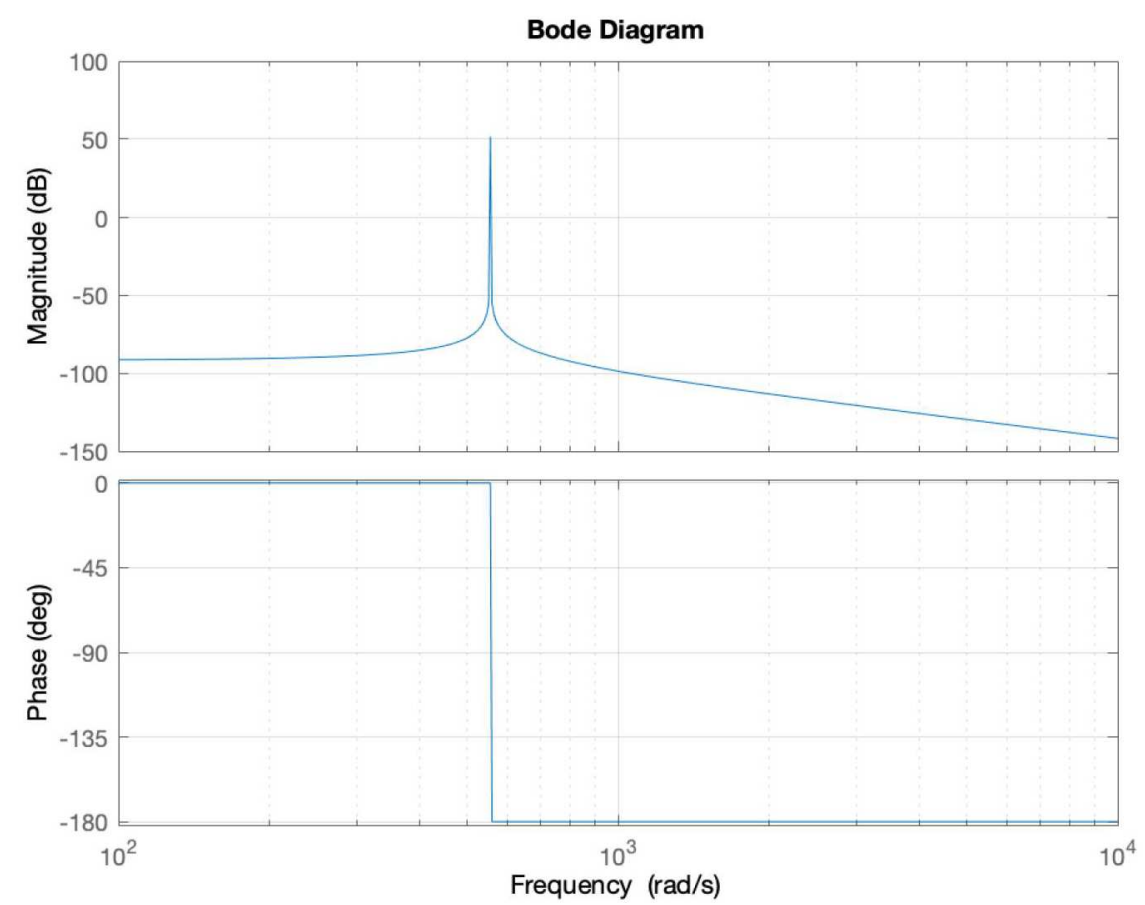

Rys. 21. Bode diagram of the transfer function

Comparing Fig. 20 and Fig. 21 with little calculation between rad/s and Hz, the phase shift takes place around the same value.

\section{Simulation}

After the analysis in the frequency domain, simulation in the time domain is then conducted with the help of MATLAB program. The simulation time is set for one full stroke, and the time step for this simulation is $5 \cdot 10^{-5}$ at this point. The time functions for three Euler angles $\phi, \theta$ and $\eta$ are taken from [1] and the step component is taken away, simplified in this model to

$$
\begin{aligned}
& \phi(t)=\phi_{m} \sin (2 \pi f t) \quad \theta(t)=\theta_{m} \cos \left(2 \pi N f t+\phi_{\theta}\right)+\theta_{0} \\
& \eta(t)=\eta_{m} \sin \left(2 \pi f t+\phi_{\eta}\right)+\eta_{0}
\end{aligned}
$$

where $\phi_{m}, \theta_{m}, \eta_{m}$ are the maximum value for all three angles. $\theta_{0}$ and $\eta_{0}$ are the offset with respect to the main flapping motion, $\phi_{\theta}$ and $\phi_{\eta}$ are the offset with respect to the heaving motion (Fig. 8). 
Using the auxiliary coordinate, the velocity and acceleration term can be determined as

$$
\begin{aligned}
& v_{x^{\prime}}=r(\dot{\phi} \cos \theta \cos \eta+\dot{\theta} \sin \eta) \quad v_{y^{\prime}}=r(\dot{\theta} \cos \eta+\dot{\phi} \cos \theta \sin \eta) \\
& a_{x^{\prime}}=r[\ddot{\phi} \cos \theta+\dot{\theta}(\dot{\eta}-\dot{\phi} \sin \theta)] \cos \eta+(\ddot{\theta}-\dot{\eta} \dot{\phi} \cos \theta \sin \eta) \\
& a_{y^{\prime}}=r[\dot{\theta}(\dot{\eta}-\dot{\phi} \sin \theta)-\ddot{\phi} \cos \theta] \sin \eta+(\ddot{\theta}-\dot{\eta} \dot{\phi} \cos \theta \cos \eta)
\end{aligned}
$$

where $v_{x^{\prime}}, v_{y^{\prime}}$ are velocity components of the wing, $a_{x^{\prime}}, a_{y^{\prime}}$ are acceleration components.

As can be seen in Figs. 8, 22 and 23, the wing motion starts from the middle of the up-stroke and continues till the subsequent upstroke. The phase shifts can be clearly seen at around $0.01 \mathrm{~s}$, where the velocity terms on both the $x^{\prime}$ and $y^{\prime}$ axes tend to zero. The acceleration components are nearly their highest.
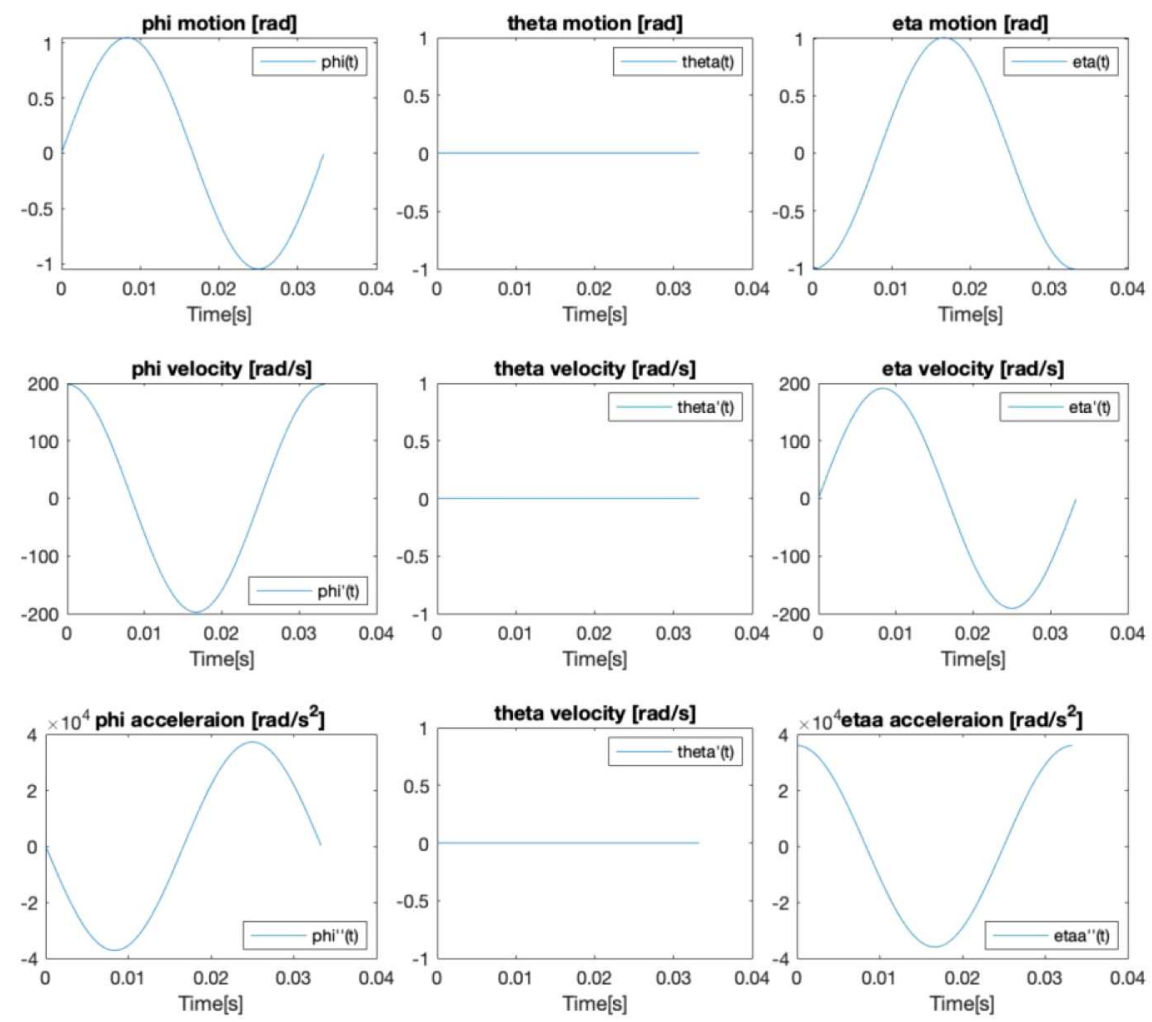

Rys. 22. Euler angles of wing motion and their velocity and acceleration terms

In Fig. 24, it can be seen that the flapping angle $\phi$ and $\eta$ are sinusoidal, the maximum value is determined by $\phi_{\max }$ and $\eta_{\max }$ and the velocity and acceleration components are very high in value. $\theta$, however, is constant zero, indicating that no out-of-plane motion is presented or considered. The effective angle of attack is modified from pitching motion $\eta$, set to be starting from $30^{\circ}$. The dynamic model used in the simulation is based on the work [1]. According to it, the coordinate is changed back to a cartesian coordinate, then the velocity components $v_{x}, v_{y}$ and the acceleration $a_{x}, a_{y}$ can determined from $\phi, \eta$ and $\theta$.

Once again, for simplification, the wing cross-section area along the chord is seen as elliptical, wing thickness is constant and the chord length is calculated on the assumption from [5]. The chord length $c(r)$ is given as

$$
c(r)=\frac{4 \bar{c}}{\pi} \sqrt{1-\frac{r^{2}}{R^{2}}}
$$



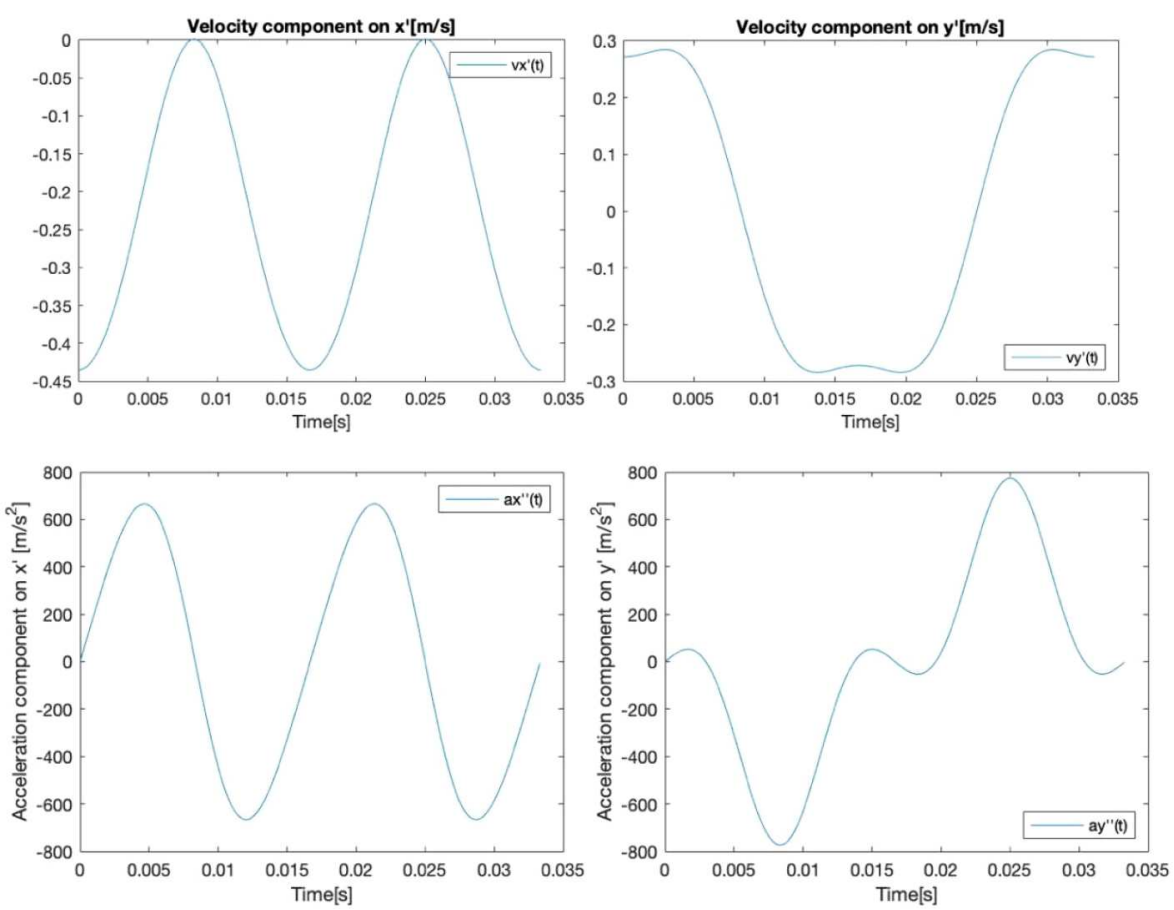

Rys. 23. Velocity and acceleration components, simulation results
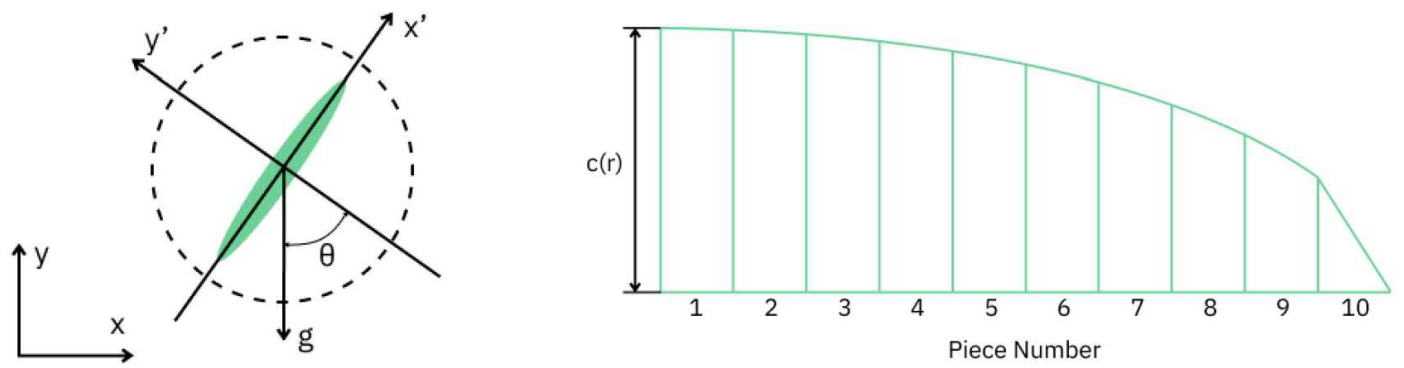

Rys. 24. The auxiliary coordinate (left) and wing strips, half view (right)

The wing is divided into 10 strips from the wing tip too the wing root in the span-wise direction, as shown in Fig. 24. It is described by width coordinates and mass of each strip. Subsequently, the inertia of the wing $I_{w} \approx I$ is obtained.

The aerodynamic forces are calculated in each strip. The quasi-two-dimensional force model made in [1] is applied. Direct calculation of the force and moment in each strip is carried out by the following equations

$$
\begin{aligned}
& d F_{x^{\prime}}=\left[\left(\frac{c(r)}{\bar{c} R} m_{w}+m_{22}\right) v_{y^{\prime}} \dot{\eta}-\rho_{a} \Gamma v_{y^{\prime}}-m_{11} a_{x^{\prime}}\right] d r-d F_{x^{\prime}}^{v} \\
& d F_{y^{\prime}}=\left[-\left(\frac{c(r)}{\bar{c} R} m_{w}+m_{11}\right) v_{x^{\prime}} \dot{\eta}-\rho_{a} \Gamma v_{x^{\prime}}-m_{22} a_{y^{\prime}}\right] d r-d F_{y^{\prime}}^{v} \\
& d_{\eta}^{\text {aero }}=\left[\left(m_{11}-m_{22}\right) v_{x^{\prime}} v_{y^{\prime}}-I_{a} \ddot{\eta}\right] d r-d \tau^{v}
\end{aligned}
$$

where $m_{11}, m_{22}$ and $I_{a}$ are the added mass terms, $\Gamma$ is the circulation around the wing, $d F_{x^{\prime}}^{v}$, $d F_{y^{\prime}}^{v}$ and $d \tau^{v}$ represent the viscous force and moment in each segment, in particular

$$
\begin{aligned}
& \Gamma=-\frac{1}{2} C_{T} c(r)|v| \sin 2 \alpha+\frac{1}{2} C_{R} c^{2}(r) \dot{\eta} \\
& F^{v}=\frac{1}{2} \rho_{a} c(r)\left(C_{D}(0) \cos ^{2} \alpha+C_{D}\left(\frac{\pi}{2}\right) \sin ^{2} \alpha\right)|v|\left\langle v_{x^{\prime}}, v_{y^{\prime}}\right\rangle d r
\end{aligned}
$$




$$
\begin{aligned}
& d \tau^{v}=\frac{1}{16} \pi \rho_{a} \rho c^{4}(r)\left[\mu_{1} f+\mu_{2} \dot{\eta}\right] \dot{\eta} d r \\
& m_{11}=\frac{1}{4} \pi \rho_{a} b^{2} \quad m_{22}=\frac{1}{4} \pi \rho_{a} c^{2}(r) \quad I_{a}=\frac{1}{128} \pi \rho_{a}\left[c^{2}(r)+b^{2}\right]^{2}
\end{aligned}
$$

where $C_{T}$ and $C_{R}$ are, respectively, translational and rotational lift coefficients, $\alpha$ is the effective angle of attack, $C_{D}(\alpha)$ is the drag coefficient of the whole wing as a function of the angle od attack $\alpha, \mu_{1}$ and $\mu_{2}$ are non-directional coefficients of fluid viscosity.

The circulation and viscosity terms around each strip through time are calculated and presented in Fig. 25.
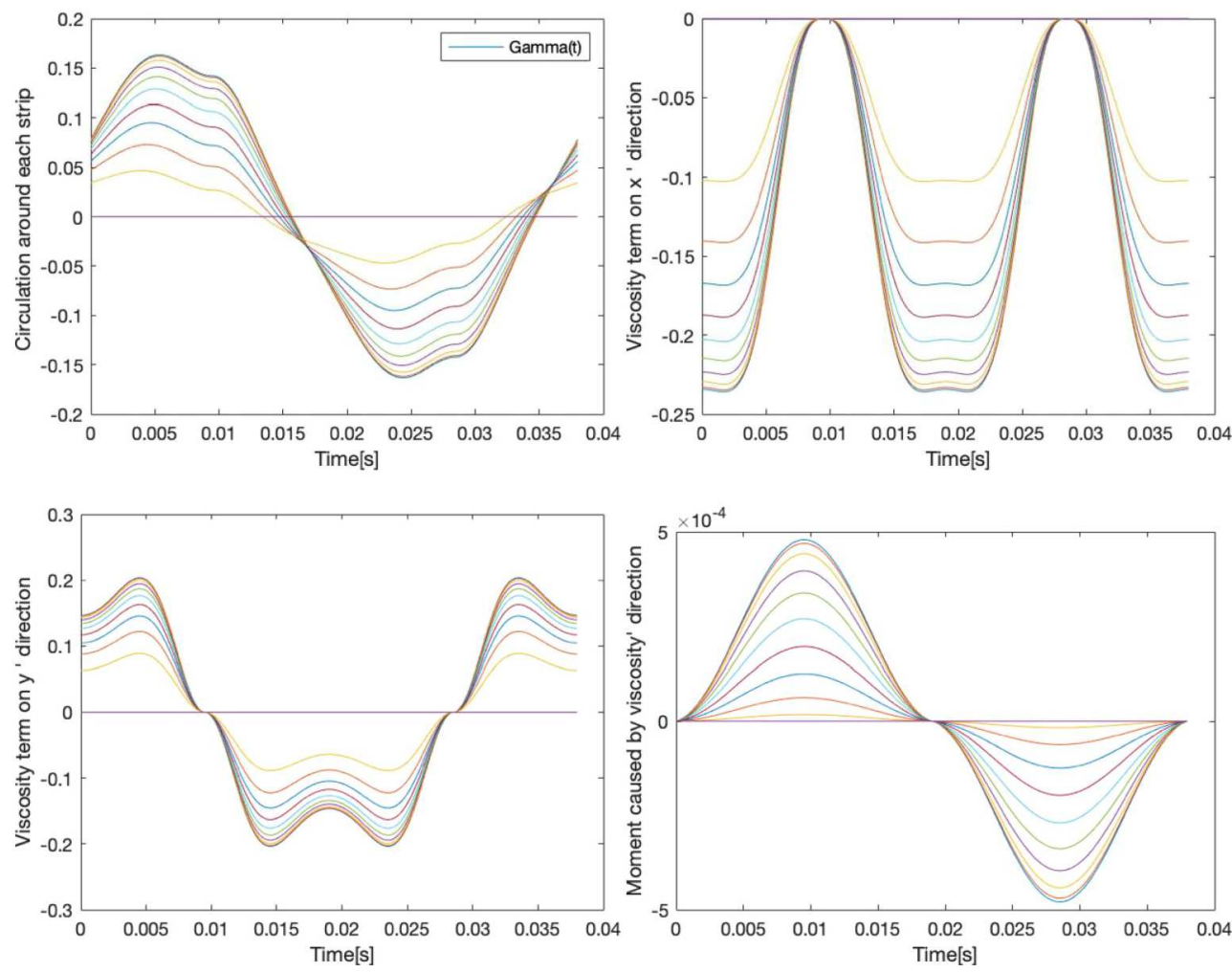

Rys. 25. Circulation and viscosity terms in each strip during one full stroke

After obtaining all essential components for the aerodynamic force and combining them all, the resulting $d F_{x^{\prime}}$ and $d F_{y^{\prime}}$ can be shown in Figs. 26 and 27.
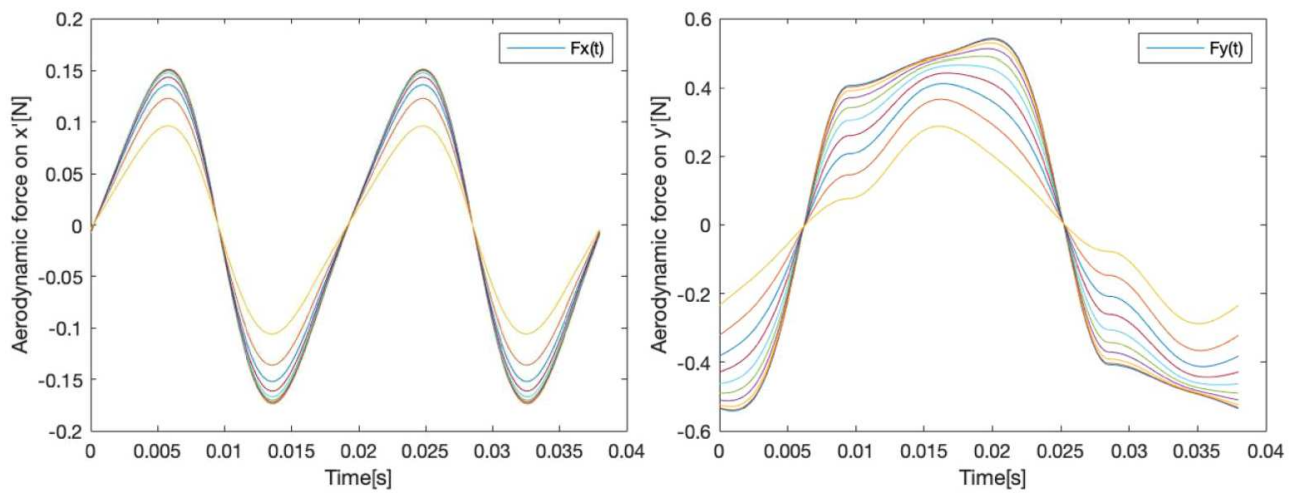

Rys. 26. Aerodynamic force for each strip in the $x^{\prime}$ and $y^{\prime}$ auxiliary coordinates 

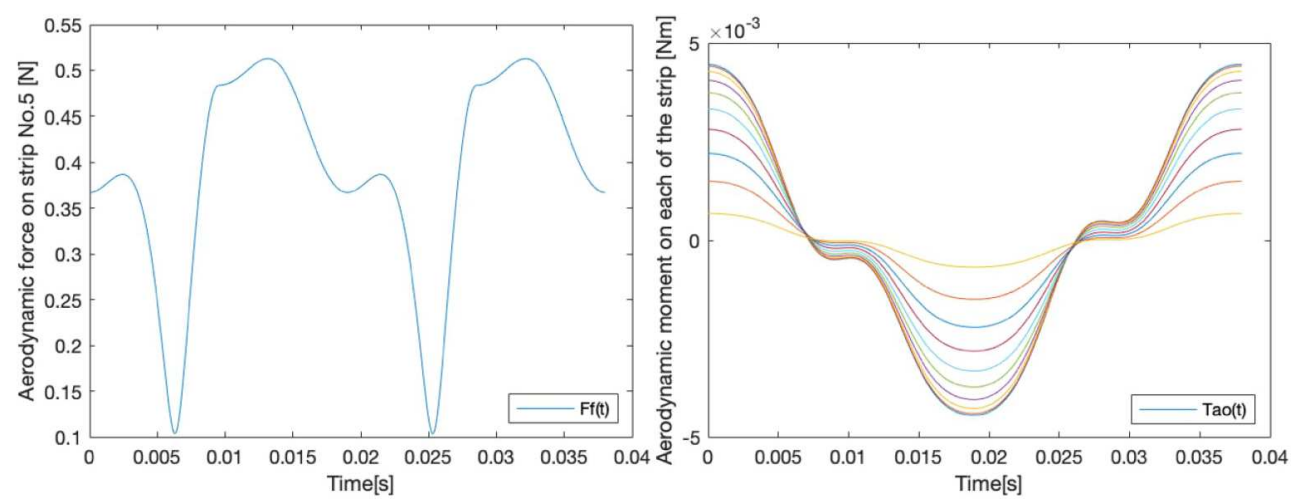

Rys. 27. Total aerodynamic force in strip No. 5 and the aerodynamic moment in each strip

From the aerodynamic force component on $x^{\prime}$ and $y^{\prime}$ axis, it can be seen that shape of the aerodynamic force through time is similar to the wing velocity component. Also, the total aerodynamic force and moment are also shown in Fig. 28.
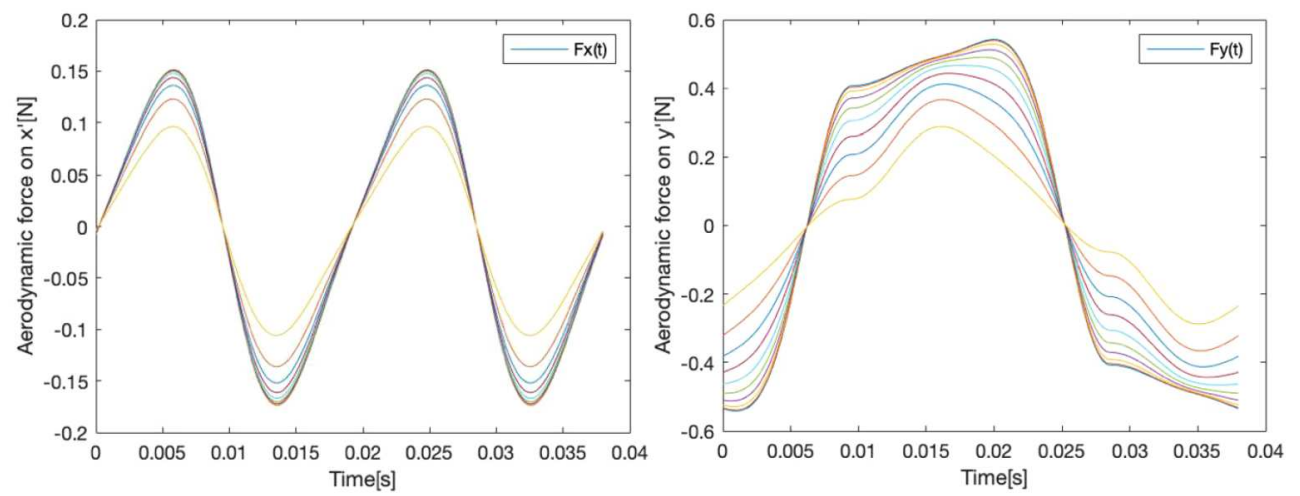

Rys. 28. Aerodynamic forces for each strip in the $x^{\prime}$ (left) and $y^{\prime}$ (right) auxiliary coordinates

With the obtained aerodynamic forces in each strip, the total forces and moments can be calculated as well converting all the forces and moments back to the $x$ and $y$ axes. They are presented in Fig. 29.
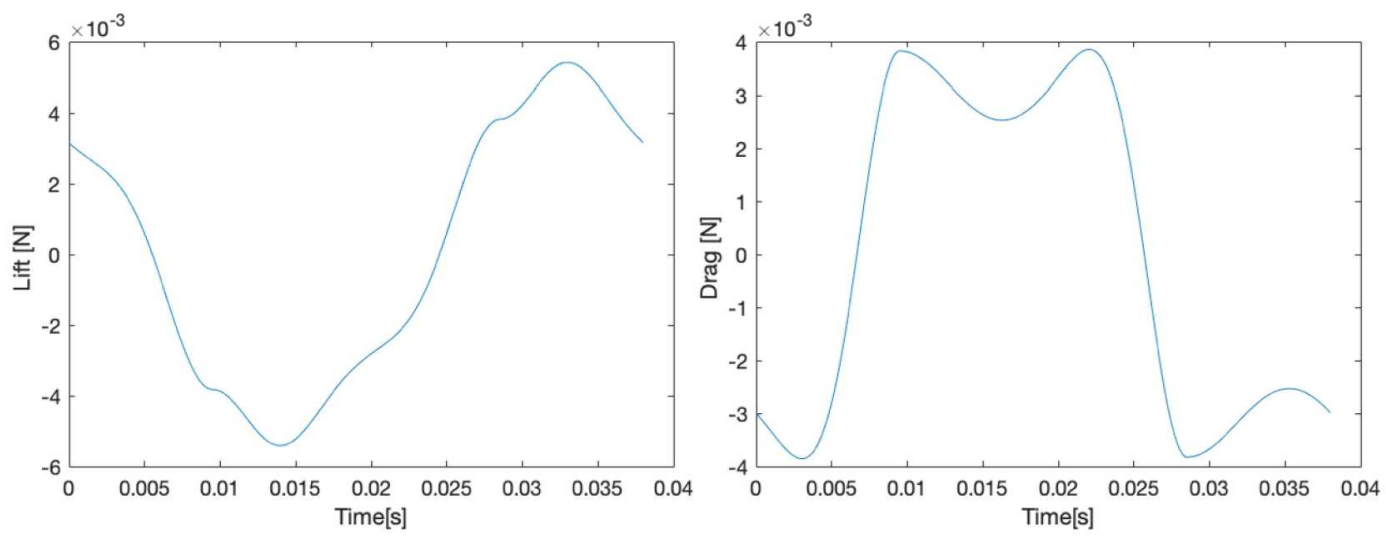

Rys. 29. Total lift and drag

The power requirement and the actuator torque are shown in Fig. 30. In these two simulations, two conditions are compared. The red line shows the situation where the stiffness term is taken out from the equation, and the blue line with it taken into account. 

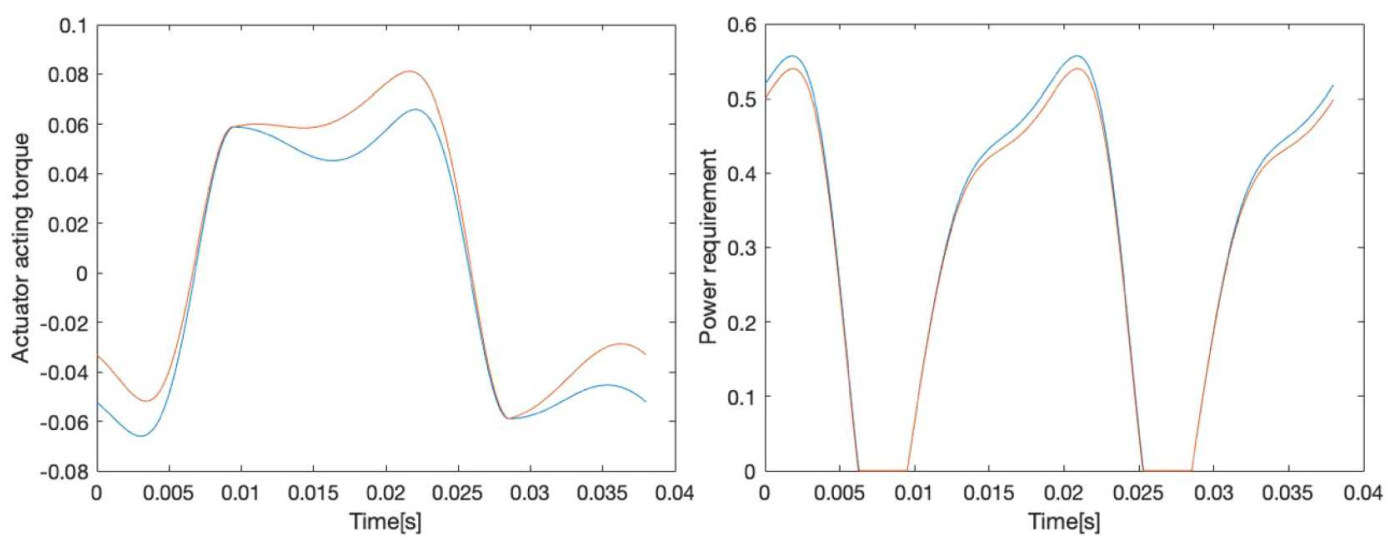

Rys. 30. Actuator torque and power requirement for including and excluding the stiffness term

For the actuator torque, from the comparisons, it can be seen that after including the stiffness term, the torque has a tendency to a sinusoidal shape and, at the same time, the torque requirement is increased. The total power consumption line is not continuous because all values lower than zero, which are not physical, are corrected to zero. The total power consumption along the red line with stiffness taken into consideration is $3 \%$ less than that without stiffness taken into consideration.

\section{Conclusions}

In conclusion, the aerodynamic forces are found to be really similar to the pattern of the pitching and flapping angle. The added mass terms, LEV and rotational circulation, dominate lift generation. By adding the stiffness term into the total equation, the power is reduced, however the actuating force requirement increases.

In future studies, the actuation and compliance are recommended to be investigated. In this paper, these aspects are not concerned for simplicity. When considering the real model, the power consumption necessary for the mechanism is really small, but the power required for the actuator to work under such a frequency can be huge.

\section{References}

1. Berman G.J., Wang Z.J., Energy-minimizing kinematics in hovering insect flight, Journal of Fluid Mechanics, 582, 153-168, 2007

2. Bolsman C.T., Flapping Wing Actuation Using Resonant Compliant Mechanisms, PhD Dissertation, Delft TU, 2010, ISBN 978-90-9025685-6

3. Ellington C., 1999. The novel aerodynamics of insect flight: application to micro-air vehicles, Jounal of Experiment Biology, 202, 3439-3448, 1999

4. Galiński C., ŻBikowski R., Materials challenges in the design of an insect-like flapping wing mechanism based on a four-bar linkage, Materials and Design, 28, 3, 783-796, 2007

5. Kolomenskiy D., Moffatt H., Farge M., Schneider K., The lighthill-Weis-Fogh clap-fling-sweep mechanism revisited, Journal of Fluid Mechanics, 676, 572-606, 2011

6. Klowden M.J., Locomotor Systems, Physiological Systems in Insects, 3rd ed., Academic Press-Elsevier Inc., Amsterdam-Tokyo, 475-527, 2013, ISBN 978-0-12-415819-1

7. Koopmans J., Delfly Freeflight Autonomous Flight of the Delfly in the Wind Tunnel Using Low-Cost Sensors, Delft TU, MSc Thesis, 2012 
8. Nan Y., Peng B., Chen Y., McGlinchey D., From studying real hummingbirds to designing hummingbird-like robots - a literature review, IEEE Access, 7, 131785-131804, 2019

9. Peters H., The Optimization of the Flapping Wings for a Micro Air Vehicle, Delft University of technology, MSc Thesis, 2011, http://resolver.tudelft.nl/uuid:f42d9d82-ed7f-450d-9c5b$7 \mathrm{~d} 34 \mathrm{~d} 3 \mathrm{ae} 2725$

10. QI W., Modeling, Design, and Optimization of Flapping Wings for Eficient Hovering Flight, PhD Dissertation, Delft TU, 2017, ISBN: 978-94-92516-57-2

11. SAnE S.P., The aerodynamics of insect flight, Journal of Experimental Biology, 206, 4191-4208, 2003, DOI: $10.1242 /$ jeb.00663

12. Truong van T., Debiasi M., Kureemun U., Lee H.P., Structural optimization of a flapping wing micro air vehicle, Yth International Conference on Mechanical and Aerospace Engineering (ICMAE), London, 476-479, 2016, DOI: 10.1109/ICMAE.2016.7549587

13. Walker S.M., Thomas A.L.R., TAylor G.K., Operation of the alula as an indicator of gear change in hoverflies, Journal of Royal Society Interface, 9, 1194-1207, 2012, DOI: $10.1098 /$ rsif. 2011.0617

\section{Analiza właściwości rezonansowego napędu skrzydeł entomoptera}

Praca jest poświęcona badaniu i zrozumieniu właściwości rezonansowego napędu skrzydeł entomoptera. Praca jest podzielona na dwie części. W części pierwszej zostały podane podstawowe informacje o owadach i trzepoczących skrzydłach entomopterów (miniaturowych bezzałogowych statków powietrznych wzorowanych na budowie latających owadów). W drugiej części opisano model matematyczny entomoptera ze szczególnym uwzględnieniem bionicznego, rezonansowego napędu skrzydeł. Opracowano program symulacyjny napisany w środowisku MATLAB i przedstawiono wyniki symulacji. 